\title{
Voces y escritura: \\ La reflexividad en el texto etnográfico
}

\author{
CRISTINA SÁNCHEZ CARRETERO \\ Instituto de Estudios Norteamericanos \\ Universidad de Alcalá
}

\section{RESUMEN}

Este artículo examina los problemas que surgen del paso del trabajo de campo a la elaboración de una monografía etnográfica. En concreto, las decisiones que toma la etnógrafa a la hora de encarar el proceso de escritura: qué voces se oirán en su trabajo, cómo aparecerán esas voces, cuál es su propia voz y qué relaciones se establecen entre su voz y las de sus colaboradores/informantes. Después de esbozar los cambios que ha habido en la antropología en los últimos treinta años, se ilustra la necesidad de introducir reflexivamente el yo de la etnógrafa en las monografías con un ejemplo del trabajo de campo de la autora en la República Dominicana.

Palabras clave: Reflexividad, Escritura etnográfica, República Dominicana, Métodos de trabajo en antropología.

\section{SUMMARY}

This article examines the problems that arise when the ethnogapher moves from fieldwork into the writing process. In particular, it explores the act of decision making before starting to write: which voices will be heard, how are these voices going to appear, which one is the ethnographer's own voice, and what are the relationships between her voice and her collaborators'. After an overview of the changes in anthropology during the last thirty years, the article points out the necessity of introducing the self in the writing process using an example from the author's fieldwork in the Dominican Republic.

Key words: Reflexivity, Ethnographic Writing, The Dominican Republic, Anthropological Methods.

La escritura de una monografía etnográfica plantea muchos problemas que tienen que ver, en parte, con la creación del sujeto de estudio, las bases que han guiado el trabajo de campo previo y la negociación que el etnógrafo hace para dar coherencia a su labor mediadora entre los

RDTP, LVIII, 1 (2003): 71-84 
procesos culturales que pretende analizar, su forma de acceder a las fuentes que le permitirán conocer/participar de dichos procesos y el análisis de los mismos. El problema antropológico del que voy a hablar en este artículo es el paso del trabajo de campo a la producción de una monografía etnográfica. En particular, la forma en la que el etnógrafo decide qué voces se oirán en su trabajo, cómo aparecen esas voces, cuál es su propia voz y qué relaciones se establecen entre su voz y las de sus colaboradores/informantes. Las voces que aparecen están estrechamente relacionadas con las que se silencian: voz y silencio (o invisibilidad, si llevamos la metáfora al sentido de la vista) son elementos que hay que tener en cuenta al encarar el proceso de escritura, aunque muchas veces este proceso de selección no se realiza de forma explícita en el producto final que llega al lector de la monografía.

Para hablar de este tema, voy a esbozar una serie de cambios que ha habido en la antropología en los últimos treinta años, tanto en la conceptualización del trabajo de campo, como en su relación con el otro (y, por supuesto, la crisis de representación de la antropología, lo que se ha venido llamando la crítica postmoderna), ilustrando la necesidad de introducir reflexivamente la participación del etnógrafo en la elaboración de su sujeto de estudio con un ejemplo de mi trabajo de campo en la República Dominicana.

La etnografía en sí misma lleva implícito el proceso de escritura, y el estatus del antropólogo como autor hace tiempo que se ha venido poniendo de manifiesto. Cuando en 1984 se reúne un grupo de antropólogos para realizar un seminario en Santa Fe coordinado por James Clifford y George Marcus sobre el acto de escritura que implica la producción de monografías etnográficas, se parte de los intentos experimentales que ya habían tenido lugar dentro de la antropología. Esta revisión textual (publicada en forma de libro dos años después como Writing Culture, o Retóricas de la antropología en español) nace de la crítica postmoderna de la antropología y empieza deconstruyendo las monografías más emblemáticas de la disciplina: Argonauts of the Western Pacific (Malinowski 1922), los estudios de Margaret Mead en Samoa (Coming of Age in Samoa, 1923) o los de Evans-Pritchard con los nuer (Nuer Religion, 1956; Witchcraft, Oracles, and Magic among the Azande, 1937)1. Nunca antes

\footnotetext{
${ }^{1}$ Como también hará, años más tarde, Clifford Geertz en El antropólogo como autor (1988). Los cuatro autores que analiza Geertz son: Claude Lévi-Strauss, Edward EvansPritchard, Bronislaw Malinowski y Ruth Benedict. Sin embargo, hay que diferenciar hacer metaantropología - es decir el análisis textual de otras monografías etnográficasde la elaboración de textos antropológicos postmodernos y Clifford Geertz no se puede considerar un autor postmoderno.
} 
se había criticado con un análisis textual tan sofisticado el hecho de que se asumiera que las etnografías eran "espejos transparentes de la cultura" (Behar 1995: 4) y se fue analizando la forma en que se construye el discurso del antropólogo, las metáforas que emplea, las relaciones de poder que entran en juego, así como los estereotipos que perpetúa. Un libro clave para que se provocara esta crisis de representación fue la publicación de los diarios de Malinowski en 1967 (traducidos del polaco al inglés). Su discípulo Raymond Firth tuvo la desagradable tarea de escribir la introducción, lo que queda reflejado en sus palabras, donde señala que el lector puede encontrar elementos de brutalidad, incluso degradantes... pero que antes de juzgar, debe ser honesto con sus propios pensamientos (Geertz 1988: 76).

A la vez que tuvo lugar esta crisis de representación, las monografías intentaban experimentar con nuevas formas de escritura y la práctica del trabajo de campo también sufrió importantes cambios, originados, en parte, por los trabajos teóricos dedicados al tema. Fue decisiva la influencia del libro editado por George Stocking, Observers Observed (1983) y su posterior obra The Ethnographer's Magic (1992) que incluye su artículo centrado en el trabajo de campo en la antropología británica desde Tylor a Malinowski, ya publicado en la colección de 1983, donde analiza el trabajo de campo como el rito de paso para llegar a ser antropólogo, y señala Los Argonautas del Pacífico Occidental de Malinowski como la carta fundacional. El arquetipo del antropólogo construido a partir de Malinowski correspondería al hombre blanco que pasa un año o más entre "nativos".

El trabajo de campo se caracterizaba, tradicionalmente, por la estancia prolongada de un investigador en una comunidad pequeña con la que no compartía la misma cultura, interfiriendo de forma mínima en la recopilación de datos. Se actuaba como si el investigador se encontrara en un laboratorio natural, donde el nativo se hallaba en un medio controlado de límites definidos. Pero en la actualidad no se pueden seguir manteniendo estas premisas y los límites claramente marcados a la hora de confrontar al otro se han ido difuminado.

La aceptación, por parte del etnógrafo, de que existe una negociación y una construcción de su yo durante el trabajo de campo, con su consiguiente reflejo en la monografía etnográfica, implica el comienzo del giro reflexivo. La presencia explícita del cómo se ha negociado el yo en el trabajo de campo aparece, por tanto, como un elemento de las monografías que incorporan la reflexividad. Esta preocupación metodológica, si bien, no tiene por qué ser el centro de la monografía - ni es deseable que lo sea- no puede obviarse después de la crisis de representación sufrida por la antropología. La interacción del yo del etnógrafo es una parte 
esencial en el trabajo de campo y no se puede pretender engañar al lector, simplemente pasándola por alto con una pretendida neutralidad en un discurso que intenta describir las cosas "exactamente como son".

La observación participante implica entenderse con una variedad de personas. Las particularidades de los informantes (colaboradores) se suelen perder o se generalizan en las monografías que presentan al grupo del que se habla bajo la autoridad suprema del autor. También es cierto que en muchas ocasiones, no son sólo las voces de los colaboradores las que se silencian, sino la propia voz del autor. El proceso de escritura y de creación del texto final, lleva consigo una serie de elecciones que dependen de los intereses del etnógrafo (Okely y Callaway 1992: xi). En el ámbito norteamericano la publicación de Retóricas de la antropología en 1986 supuso una llamada de atención sobre nuevas formas de escritura. En el mundo británico, Edmund Leach sugería en 1987, en la reunión anual de la Asociación de Antropólogos Sociales, que "los etnógrafos deben admitir la reflexividad de sus actividades" (citado por Okely y Callaway 1992: xii).

Esta reflexividad se halla en varios niveles. Desde la presencia del investigador durante su trabajo de campo a las reacciones de los informantes o la influencia de las lecturas en las orientaciones teóricas del investigador (Davies 1999: 229). Sin embargo, también ha habido críticas al giro reflexivo, en ocasiones calificándolo de "narcisista" o de "auto-adoración". Pero tener conciencia de uno mismo no implica -como superficialmente suelen criticar los que apenas han leído monografías de este tipo- que la escritura del antropólogo se transforme en un acto de exhibicionismo. Estas críticas tienen su base en la nostalgia de un único paradigma. La pasión por el todo ha marcado a la antropología hasta hace poco y la multitud de paradigmas actuales, tal vez moleste a los que han venido desarrollando su carrera siempre en un mismo paradigma. Sin embargo, las "verdades parciales" de las que habla James Clifford, pueden ayudar a asumir el carácter incompleto de las etnografías (Clifford 1986: 1)2.

En el campo concreto de los estudios de folklore, las transformaciones que ha habido en el trabajo de campo y su paso a la forma escrita han cambiado drásticamente desde los primeros "recolectores de curiosi-

\footnotetext{
${ }^{2}$ Además de las monografías reflexivas, desde los años cincuenta hay abundantes autobiografías escritas por antropológos. Véanse, por ejemplo las de Laura Bohannan, Return to Laughter (1954); Hortense Powdermaker, Stranger and Friend (1967) o Paul Rabinow, Reflections on Fieldwork in Morocco (1977). Las descripciones autobiográficas "auto-reflexivas" se pueden considerar como un particular género etnográfico (Stocking 1992: 13). Dentro del ámbito europeo, Okely (1992: 11) señala los trabajos de FavretSaada (1980), Favret-Saada y Contreras (1981) y Loizos (1981) como ejemplos de monografías etnográficas en formato autobiográfico.
} 
dades" que con carácter amateur se dedicaban a la recogida de textos motivados por una búsqueda romántica de un pasado idealizado (en otros casos, había fines mucho más siniestros, como el caso de los institutos de Volkskunde en la Alemania nazi). Hasta mediados del siglo XX, la finalidad era la recogida de textos, tanto para los investigadores que desarrollaban su trabajo en instituciones académicas, como para los que lo hacían como actividad de ocio. A partir de los años sesenta los estudiosos del folklore empezaron a centrar su atención en el contexto y estos cambios en el trabajo de campo supusieron que el papel del investigador variara de "recolector" a "observador participante". Al abrirse esta puerta, el giro contextual hizo moverse los estudios de folklore de la recogida de textos desarticulados a dar una mayor importancia a los procesos creativos, performativos y de uso que realizaban personas concretas.

El cambio de paradigma quedó plasmado, en Estados Unidos, en 1972, con la publicación del libro Toward New Perspectives in Folklore (editado por Américo Paredes y Richard Bauman) que sintetizaba los nuevos puntos de vista, prestando especial atención a la base social y la creación diferencial de las identidades. Al entender el folklore como proceso, se daba paso a todo tipo de aspectos políticos, de invención de la tradición y la comercialización de la cultura. Las propias críticas textuales de las que hablábamos al principio dieron lugar a nuevas historias de esta disciplina, reconociéndose el propio encuentro etnográfico como una performance (Briggs 1993) ${ }^{3}$.

Por un momento voy a centrarme en el trabajo de Américo Paredes como pionero en los estudios de minorías en los Estados Unidos, porque puede servir de ejemplo a las discusiones sobre el antropólogo "en casa". Desarrolló el concepto de frontera como lugar de convergencia, conflicto y creatividad cultural, centrándose en las tensiones culturales que él observaba desde su experiencia de haber crecido en la frontera entre México y Texas. Américo Paredes después de haber estudiado durante varios años su propio grupo, dedica su artículo "El trabajo etnográfico entre grupos de minorías" (1977) a las relaciones de poder que se negocian en las dimensiones performativas del encuentro etnográfico. Paredes señala los problemas que encuentra cuando los anglo-americanos estudian la cultura de los mexicano-americanos, señalando que estos investigadores suelen: (1) reflejar los estereotipos que tienen sobre los mexicano-americanos los pertenecientes a la cultura dominante; (2) no están familiarizados con el uso del folklore como herramienta en luchas sociales y políticas; y (3) no se dan cuenta de las múltiples maneras en las que se pueden comunicar diferentes significados (Stoeltje, Fox y Olbrys 1999: 169-170).

\footnotetext{
3 Véase Dorothy Noyes (2000: 28-29).
} 
Al poner el dedo en la llaga en las relaciones que se establecen en el encuentro etnográfico, saca a la luz un hecho obvio, pero que sin embargo se suele seguir pasando por alto: el encuentro etnográfico es un acto comunicativo mucho más complejo de lo que admitimos y que hay múltiples dimensiones de las interacciones que se producen, por ejemplo, una de ellas sería que los discursos de nuestros colaboradores pueden surgir de nuestras propias motivaciones. El primer paso que Américo Paredes sugiere para mejorar el método etnográfico es rechazar la idea de comunidad como algo estático y homogéneo en donde las personas son vistas como unidades intercambiables, abogando por el respeto a cada uno de los informantes con sus particularidades, lo que no implica que no se puedan sacar conclusiones generalizables.

Antes de comenzar el proyecto etnográfico, el investigador ya se ha planteado, por supuesto, aspectos de contenido, como "qué problema queremos analizar", y cuestiones metodológicas y prácticas, por ejemplo "cómo vamos a escoger a nuestros colaboradores", "qué permisos hay que pedir" o "cómo vamos a iniciar los contactos" - aspectos que van más allá del objetivo de este artículo. Tampoco voy a ahondar en técnicas antropológicas como la observación participante, la entrevista en profundidad, entrevistas guiadas... etc, sino en los objetos de los que se parte para escribir textos etnográficos. En gran medida, estos materiales se utilizan para dar autenticidad al texto que se produce demostrando que el etnógrafo ha estado allí (Davies 1999: 119), también tienen función de ilustrar lo que se describe y analiza (tanto si se incorpora o no al texto), pero pueden dar pie a un análisis en sí mismos.

Los materiales que el etnógrafo va acumulando, es decir, los que van a formar las fuentes primarias de la investigación etnográfica se suelen dividir entre los que se consiguen durante le trabajo de campo y los que se gestan en la mesa de trabajo (Velasco y Díaz de Rada 1997). Yo me voy a referir a todos a la vez, porque lo que me interesa señalar es su base textual. Cada vez hay una variedad de instrumentos de registro mayor, sin que haya acuerdo sobre qué tipo de anotaciones son más útiles, cuándo se deben escribir y si limitan el análisis de lo observado, o si se deben separar drásticamente las anotaciones sobre lo observado y las ideas del observador. Estos problemas deben ser resueltos por el etnógrafo, pero sin olvidar que hay toda una experiencia anterior de la que podemos aprender ${ }^{4}$.

\footnotetext{
${ }^{4} \mathrm{La}$ imagen del antropólogo autosuficiente ha sido descrita como el "síndrome de Indiana Jones" (término usado por Jean Jackson y citado por Roger Sanjek 1990: 92), para criticar que, utilizando la excusa de que la experiencia va forjando al antropológo, se pasen por alto las diferentes técnicas de trabajo de campo.
} 
Entre los materiales que se generan durante el trabajo de campo cabe destacar ${ }^{5}$ :

1. Textos que produce el etnógrafo: cuadernos de notas, diario, cuaderno de campo y todo tipo de notas y observaciones.

2. Textos escritos por "los otros": cartas, diarios, todo tipo de materiales de archivos, periódicos, documentos de instituciones, etc.

3. Objetos de la cultura material del lugar.

4. Grabaciones de audio/vídeo ${ }^{6}$.

5. Fotografías.

6. Nosotros mismos, es decir, nuestras notas mentales.

Un tipo de notas que los etnógrafos escriben son las anotaciones in situ: breves frases, palabras, esquemas que servirán para fijar observaciones, se llaman "cuadernos de notas" (scratch notes). En los cuadernos de campo (o "diario de campo" según Velasco y Díaz de Rada 1997: 92) 7 se produce el paso de las notas tomadas in situ mediante el proceso que Clifford Geertz llama "inscripción". Este concepto ha sufrido muchas críticas y se han utilizado otros como "transcripción", "traducción" (en el sentido de traducir una cultura en terminos de otra), o "textualización", es decir, transformar experiencias en textos (Emerson, Fretz y Shaw 1995: 15). Los cuadernos de campo están escritos para el propio etnógrafo, para que interactúen con sus "notas mentales" (término de Ottenberg, citado por Sanjek 1990: 93). Mientras que las notas que se escriben permanecen estáticas, las mentales van cambiando y durante toda la vida del etnógrafo, suelen ser las principales en su escritura. A la hora de repasar las fuentes en las que el etnógrafo basa su análisis, se suele pasar por alto las notas mentales que se van entremezclando con los diferentes materiales etnográficos y sin embargo son una pieza clave para entender nuestro

\footnotetext{
${ }^{5}$ Para un estudio exhaustivo de la estructura de un proyecto etnográfico véase la obra de Honorio Velasco y Ángel Díaz de Rada, La lógica de la investigación etnográfica (1997). Los trabajos de Robert M. Emerson, Rachel I. Fretz y Linda L. Shaw (1995) sobre la escritura de cuadernos de campo, incluyen muchos ejemplos y son una excelente herramienta a la hora de enseñar técnicas de trabajo de campo; así mismo, el clásico libro de Bruce Jackson (1987) utiliza un tono conversacional con muchos trucos prácticos a la hora de encarar un proyecto sobre cultura expresiva.

${ }^{6}$ Los cambios en la tecnología han marcado el camino de la evolución en el trabajo de campo. Véase Edward D. Ives (1990) y, sobre todo, Erika Brady (1999). Brady analiza el modo en que el fonógrafo cambió la forma de realizar etnografía.

7 Pienso que es preferible reservar la palabra "diario" para las anotaciones más personales.
} 
trabajo. Otros textos producidos por el etnógrafo son los diarios personales -normalmente escritos para uno mismo con una finalidad de catarsis $^{8}$-, las cartas y los informes que escribe sobre su proyecto. A su vez, todos estos tipos de fuentes escritas que genera el etnógrafo, pueden hacerse sobre los textos escritos por los otros (materiales de archivos, cartas, diarios, periódicos). Por supuesto, otro nivel textual estaría formado por los materiales que genera el etnógrafo a partir de todo tipo de fuentes secundarias y que va influyendo en la forma de observar y escribir los cuadernos de campo (y también genera más notas mentales).

El trabajo de campo se puede realizar sobre esos materiales producidos por los otros, por lo que ha dejado de ser un fetiche y ha incorporado también el trabajo en archivos, lectura de periódicos, documentos de gobierno, internet, análisis de discursos públicos, incluso, obras de ficción. Del mismo modo, desde hace ya más de treinta años, no se discute que se puede realizar trabajo antropológico "en casa". El trabajo de campo, por tanto, no implica - necesariamente- un cambio de lugar, sino un cambio de actitud hacia lo que se observa.

Hasta finales de los años setenta (antes del giro reflexivo), la mayoría de las monografías etnográficas optaban por un narrador omnisciente. El yo del autor, sólo se usaba en contadas ocasiones para dar autoridad al texto, dejando lo personal para otro género de escritura dentro de la práctica antropológica: el diario?. Normalmente, el yo del etnógrafo, en estas monografía aparece en la introducción para explicar las circunstancias del trabajo de campo (sobre todo la llegada), pero luego se diluye y, de vez en cuando, vuelve a salir a la superficie en el epílogo. Es decir, se incluye como un elemento del contexto, sin que se analicen las particularidades de creación del sujeto de estudio (Fabian 1983). El próposito suele ser justificar la autenticidad de lo que se cuenta, justificar que se ha estado allí. Es el being there de Geertz: al etnógrafo no se le cree por la precisión y extensión de sus descripciones, sino por su solidez como autor (Geertz 1988: 4).

De una voz única en los estudios clásicos, en los que las palabras de los informantes se transcribían para ponerlas al servicio de la narración principal, se pasó a la aparición de múltiples voces, múltiples perspecti-

${ }^{8}$ Si bien, después de la publicación del diario de Malinowski, seguro que la autocensura está en las mentes de todos los que escriban un diario personal.

${ }^{9}$ La publicación del diario de Malinowski en 1967, sorprendió a muchos de sus seguidores, puesto que en él no seguía las propuestas metodológicas que proponía en Los Argonautas. Por ejemplo, en el diario se ve que Malinowski tenía mucho contacto con hombres blancos mientras que oficialmente proclamaba la inexistencia de trato (Okely 1992: 6-7). 
vas en diálogo con la voz del autor (y a veces enfrentadas a él) ${ }^{10}$. Para hacer referencia a la forma en la que las voces aparecen en el texto etnográfico, a veces se alude a metáforas como la de la traducción: traducir una cultura en términos de otra. Se ha hablado incluso de ventrilocuismo para designar la relación de la voz del autor con sus informantes en la etnografía: tanto la voz como los mensajes que quiere transmitir el autor se proyectan en el otro. Parece que el otro habla de forma autónoma (que tiene capacidad de actuar, de ser agente, pero el lector se puede dar cuenta del acto de ventrilocuismo). Pero también la voz del otro se puede silenciar completamente (dando lugar a su invisibilidad). O puede ser apropiada por el autor, por lo tanto los mensajes del otro ven la luz, pero su voz se silencia. Por último, también se puede personificar, individualizar, poniéndole una cara, un nombre, para que el lector reconozca que esos mensajes vienen de una fuente determinada.

En cuanto al tiempo verbal que se emplea en las monografías, el presente etnográfico ha sido ampliamente criticado como medio de distanciamiento del otro y de falsa objetividad (Fabian 1983). Paradójicamente, los mayores críticos de la forma clásica de escribir etnografías han sido también puestos en el ojo de mira ${ }^{11}$. De todas formas el "presente etnográfico" no suele ser tal, combinándose con tiempos pasados (a veces el análisis se hace en presente y las descripciones en pasado). La antropóloga danesa Kirsten Hastrup, por ejemplo, defiende un uso creativo del presente como mecanismo para preservar la realidad del encuentro etnográfico (Hastrup 1992: 117).

Las nociones de reflexividad se ven (por sus detractores) como una amenaza para los cánones de la disciplina, ya que es un ataque directo al positivismo. El yo reflexivo del etnógrafo echa por tierra la idea del observador como "máquina impersonal" (Okely 1992: 24). Al alejarse de una pretendida objetividad, el "yo" del etnógrafo tiene relevancia en todas las dimensiones de su investigación. Esta relevancia ha sido descrita por Beverly Stoeltje, Christie L. Fox y Stephen Olbrys como el círculo hermenéutico en el cual todos estamos personal, social e históricamente involucrados con lo que pretendemos conocer. Las personas que participan en investigaciones etnográficas se benefician más cuando su propio

${ }^{10}$ Por ejemplo, Ruth Finnegan en Tales of the City (1998) incluye las voces de sus informantes y las voces de otros investigadores sobre el tema en un plano de igualdad.

${ }^{11}$ John Davis habla, por ejemplo, de que Renato Rosaldo en su crítica a la forma de escribir etnografías de Evans-Pritchard, emplea los mismo tiempos y hace uso de las mismas estrategias que critica (Davis 1992: 209). 
"yo" ha sido integrado en el modelo de investigación, reconociendo su complejidad y las prácticas culturales que lleva implícito, puesto que el yo del investigador es el que entra en negociaciones (o no), con la gente que colabora en el estudio que uno realiza; y es el yo el que al final da cuenta de los datos, las representaciones y las interpretaciones teóricas del trabajo etnográfico (Stoeltje, Fox y Olbrys 1999: 179).

Después de este repaso por los materiales a partir de los cuales se comienza/continúa la parte interpretativa que se desarrollará en la monografía, llega la parte más problemática: ¿hasta qué punto incluir la reflexividad de la que hemos venido hablando? ¿cómo escoger la forma de narrar que mejor describa -0 que menos moleste-? Es un lugar común - aunque cierto- decir que no existen fórmulas, si bien una vez más reitero la necesidad de no seguir ocultando el yo del trabajador de campo. Mi investigación (todavía en curso) sobre las prácticas religiosas entre dominicanos en la diáspora, debido a la especial atención que presto a los narradores de historias y a los aspectos performativos, me ha llevado a plantearme la necesidad de introducir reflexivamente mi propio yo. Trataré de ilustrar esta necesidad con el ejemplo de una de las grabaciones de vídeo que realicé en el suroeste de la República Dominicana.

Durante varios meses asistí a reuniones de vudú dominicano en esta zona $^{12}$ y en una de las reuniones la portadora de misterios y encargada de ese centro, Dolores ${ }^{13}$, empezó a contar historias dirigidas a la etnógrafa, en concreto al vídeo de la etnógrafa. Semanalmente en casa de Dolores hay reuniones en honor a santos, también llamados misterios, luases o espíritus $^{14}$. Estos encuentros suelen comenzar rezando un rosario y luego pasan a incluir toques de palos ${ }^{15}$. Los santos suelen poseer el cuerpo de la "portadora de misterios" o "caballo" y se dice que "el caballo está montado". Estas reuniones, que, dependiendo de la finalidad y la duración, se llaman horas santas o veladas (o "velaciones") son un contexto habitual para narrar historias (acción que se conserva en los centros equi-

\footnotetext{
${ }^{12}$ Entre noviembre de 2000 y febrero de 2001 asistí a estas reuniones en la República Dominicana y a partir de junio de 2001 a reuniones similares en un centro de vudú dominicano en Madrid. Véase la tesis doctoral: "Narrating Diasporas: Strategies in the Creation of Locality and Agency Among Dominican Women Abroad" (SánchezCarretero 2002).

13 Utilizo pseudónimos para mis colaboradores.

${ }^{14}$ Si bien la antropóloga Martha Ellen Davis señala que los santos y los misterios no son lo mismo y que no se corresponden exactamente los santos con las deidades africanas (Davis 1987: 128), todos mis colaboradores utilizan indistintamente santo y misterio, reservando la palabra lua para los misterios de origen haitiano.

15 Los palos son un tipo de tambores.
} 
valentes que hay en Madrid). La tarde de viernes en que se narraron las historias que voy a relatar a continuación, ya habían subido ${ }^{16}$ Santiago (Ogún Balenyó), La Dolorosa (Metresilí, esposa de Santiago), y después Santa Marta (Metresa, de la división guedé ${ }^{17}$, hija del Barón del Cementerio y de "Mamá Buyita"), que es la que me interesa para el ejemplo que quiero exponer. Santa Marta había subido al cuerpo de Dolores y me dio permiso para rodar en vídeo una entrevista con ella mientras tenía lugar la hora santa, donde escenificó una historia que otros días había contado la santa para "enseñar divirtiendo"18. En esta ocasión contó que ella es del Congo y escenificó la forma en la que se convirtió en santa, para pasar a un debate teológico con una de las componentes de la hermandad, Josefa, quien cuestionó su santidad debido al desenfreno de la actividad sexual de Santa Marta ${ }^{19}$. Fue un debate intenso donde "Marta", como

${ }^{16}$ Se emplea el verbo "subir" para describir el momento en el que el misterio entra en el cuerpo de su caballo.

17 En el vudú dominicano los misterios se agrupan en divisiones, con relaciones jerárquicas entre sí: "1. Los Radás son llamados 'los Blancos' porque son los más espirituales, más positivos - no se prestan para hacer el mal y son superiores en rango [...] Son los misterios universales en los grandes cultos afroamericanos porque son loas africanos; los Legbá, los Ogón, los Arcángeles, etc. —estos seres representan aquella herencia más obviamente africana, la de Guinea [...] 2. Los Guedés son a manera de apodo 'Los Negros'. Son ambiguos, enigmáticos, porque representan la muerte y trabajan con los muertos y por lo tanto, tienen la capacidad y disponibilidad de hacer el bien o hacer el mal. Depende de quién los invoca y para cuáles propósitos. Así es el caso del culto mayombé en Cuba, también de herencia congo-angoleña, como hemos dicho trabaja con los espíritus, trabaja con magia. Los Guedés abarcan al Barón del Cementerio y sus hijos, veintiún hermanos, de los cuales hay una sola hembra, Guedé Liá (a veces escrito "Guedeliá") —Santa Marta la Dominadora. Como representan la muerte, los Guedés trabajan debajo de la tierra, en el cementerio [...] 3. Los Petrós son una división supuestamente de origen criollo y específicamente dominicano. Pero realmente parece haberse basado en gran parte de la religión bantú, que se estaba introduciendo precisamente en el siglo XVIII cuando Moreau de St. Méry notó el surgimiento del culto. Los Petrós son campestres, así manifestando la orientación animista y naturalista de la religión de la región bantú. Son los seres más 'materiales' (terrestres, mundanos) de todos, y están asociados con la magia (inclusive la magia negra o brujería) y el faquirismo —o sea, actos de sensacionalismo, como caminar encima de fuego, comer candela, pasar alfileres por la piel, beber gasolina, etc." (Davis 1987: 136-7).

${ }^{18}$ Este misterio suele contar muchas historias en las que mezcla el humor con claras provocaciones sexuales. Santa Marta se llama a sí misma "la Dominadora, la Chingadora". Según Santa Marta, para aprender sobre Dios, es mejor hacerlo riendo. Otros misterios como Metresilí o Santiago son mucho más serios y recatados.

${ }^{19}$ Según Martha Ellen Davis, Santa Marta no habla, pero mi experiencia es muy diferente: "Guedé Liá o Santa Marta, que también limpia el ambiente de malas corrientes, es precisamente la única hija del Barón. Es decir que estos Guedés, además 
la llaman sus devotos, hizo repetir a Josefa — para ver si se enteraba de lo que decía - uno a uno sus argumentos por los que, según Marta, no estaba mal que tuviera más de un marido a la vez. Todo el público, unos veinticinco hombres y mujeres de varias edades (incluyendo a los paleros, o músicos que tocan los atabales), seguía entusiasmado el desarrollo de la polémica y la etnógrafa quería conservar imágenes de la respuesta de los asistentes, por lo que comencé a girarme para sacar a todos los participantes del evento. En ese momento Santa Marta dejó de hablar y dirigiéndose a uno de sus ayudantes dijo "ipor qué hace eso?" Manuel le intentó explicar que tenía que sacar a todos pero Santa Marta se ofendió y no continuó hasta que yo pedí disculpas. Al día siguiente me reuní en el mismo centro con Dolores y algunos de los participantes en la hora santa para ver el vídeo con ellos y grabar sus comentarios y explicaciones. Dolores estaba muy interesada en verse como Santa Marta y explicó que fue una falta de respeto hacia la santa el dejar de enfocarla ya que "ella es lo más importante en la hora santa".

Si analizamos lo ocurrido desde los diferentes niveles de público que asistieron (aunque sea en diferido) a la hora santa, entenderemos la importancia de no eliminar el vídeo que se estaba grabando como un elemento más de la performance. Cuando pedí permiso a Dolores para hacer grabaciones en vídeo para completar mi trabajo de campo, me dijo que sí pero me puso una condición. Yo esperaba que la condición fuera guardar completo anonimato de los participantes o prometer que no lo divulgaría... sin embargo su premisa fue mucho menos restrictiva: mostrárselo a su familia en España, en los bares de Aravaca y hacerle una copia de las cintas para ella. Ya antes había escuchado las catequesis irónicas con que Santa Marta amenizaba las veladas en el centro de Dolores, pero para entender la dureza de las contestaciones que dirigió a Josefa hay que tener presente el público implícito que supone la grabación de un vídeo. En este caso, la etnógrafa podría analizar la narración de la vida de Santa Marta y su performance en un centro de vudú dominicano dejando fuera el papel jugado por la cámara y los futuros públicos del vídeo. Sin embargo, tomar esta opción, es decir, no incorporar elementos reflexivos y omitir el "yo" de la etnógrafa en el proceso de escritura, eliminaría de un plumazo la riqueza de niveles performativos que entran en juego en la historia de Santa Marta.

de Dambalá, comparten el papel muy importante de realizar limpiezas de ondas y entidades negativos. Y, como estos tres son los únicos loás que se presentan como no-humanos (sino como serpientes o muertos), se pueden considerar como los luás más espirituales porque tampoco hablan" (Davis 1987: 132). 
Este artículo no pretende dar una solución unívoca ni ofrecer un modelo para incluir la polifonía que supone el encuentro etnográfico; tampoco hay atajos en la búsqueda de un equilibrio en la continua negociación entre la propia voz y la de los otros. Sin embargo, este trabajo intenta ser un breve pero firme recordatorio de la importancia de incorporar la reflexividad en las monografías etnográficas. Por muchas razones, pero sobre todo por la honestidad que debemos a los lectores. La reflexividad nos fuerza a pensar en las consecuencias de nuestras relaciones con otros, tanto si son en condiciones de reciprocidad, asimetría o de explotación potencial. "Al insertar el yo del etnógrafo como sujeto posicionado, estamos obligados a hacer frente a las responsabilidades morales y políticas de nuestras acciones" (Okely y Callaway 1992: 24), lo que abre la puerta a toda una serie de consideraciones éticas que es necesario tener en cuenta. Por lo tanto, querámoslo o no, las decisiones que tomamos a la hora de encarar la escritura y las actitudes que tenemos (conscientes o no) reflejan unas relaciones de poder que hay que explicar como parte de la monografía etnográfica.

\section{BIBLIOGRAFÍA CITADA}

BeHAR, Ruth. 1995. "Introduction: Out of Exile", en R. Behar y D. A. Gordon (eds.), Women Writing Culture: 1-29. Berkeley: University of California Press.

Brady, ERIKA. 1999. A Spiral Way: How the Phonograph Changed Ethnography. Jackson: University Press of Mississippi.

Briggs, Charles L. 1993. "Metadiscursive Practices and Scholarly Authority in Folkloristics". Journal of American Folklore 106: 387-434.

CLIFFORD, JAMES. 1986. "Introduction: Partial Truths", en J. Clifford y G. E. Marcus (eds.), Writing Culture: The Poetics and Politics of Ethnography: 1-26. Berkeley-Los Angeles: University of California Press.

- y George E. Marcus (eds.). 1986. Writing Culture: The Poetics and Politics of Ethnography. Berkeley-Los Angeles: University of California Press.

Davies, Charlotte Aull. 1999. Reflexive Etnography. A Guide to Researching Selves and Others. Londres: Routledge.

DAvis, JOHN. 1992. "Tense in Ethnography. Some Practical Considerations", en J. Okely y H. Callaway (eds.), Anthropology and Autobiography: 205-220. Londres: Routledge.

DAVIS, MARTHA Ellen. 1987. La otra ciencia. El vodú dominicano como religión y medicina populares. Santo Domingo: Editora Universitaria-UASD.

Emerson, Robert M.; RACHel I. Fretz y Linda L. SHAw. 1995. Writing Ethographic Fieldnotes. Chicago: University of Chicago Press.

Fabian, Johannes. 1983. Time and the Other: How Anthropology Makes Its Object. Nueva York: Columbia University Press.

FaVRET-SAADA, Jeanne. 1980 [1976]. Deadly Words: Witchcraft in the Bocage. Cambridge: Cambridge University Press. 
- y José Contreras. 1981. Corps pour corps: Enquête sur la sorcellerie dans le Bocage. París: Gallimard.

FINNEGAN, RUTH. 1998. Tales of the City. A Study of Narrative in Uraban Life. Cambridge: Cambridge Universty Press.

GEERTZ, ClifFord. 1988. Works and Lives. The Anthropologist as Author. Stanford: Stanford University Press.

HASTRUP, KIRSTEN. 1992. "Writing Ethnography. State of the Art", en J. Okely y H. Callaway (eds.), Anthropology and Autobiography: 116-133. Londres: Routledge.

IVES, EDWARD D. 1990 [1974]. The Tpae-Recorder Interview: A Manual for Field Workers in Folklore and Oral History. Knoxville: The University of Tennessee Press.

JAKSON, BRUCE. 1987. Fieldwork. Urbana: University of Illinois Press.

LOIZOS, PETER. 1981. The Heart Grown Bitter: A Chronicle of Cypriot War Refugees. Cambridge: Cambridge University Press.

Malinowski, Bronislaw. 1922. Argonauts of the Western Pacific: An Account of Native Enterprise and Adventure in the Archipelagoes of Melanesian New Guinea. Londres: Routledge and Sons.

NOYES, DOROTHY. 2000. "Introducción: Sociedad y estudios de folklore en USA", en C. Sánchez Carretero y D. Noyes (eds.), Performance, arte verbal y comunicación: Nuevas perspectivas en los estudios de folklore y cultura popular en USA: 19-32. Oiartzun: Sendoa.

OKely, JUdith y Helen CAllaway (eds.). 1992. Anthropology and Autobiography. Londres: Routledge.

PAREDES, AMÉRICO. 1977. "On Ethnographic Work among Minorities: A Folklorist's Perspective". New Scholar 6: 1-32.

Paredes, Américo y Richard Bauman (eds.). 1972. Toward New Perspectives in Folklore. Austin: University of Texas Press.

SÁNCHEZ CARRETERO, CRISTINA. 2002. Narrating Diasporas: Strategies in the Creation of Locality and Agency Among Dominican Women Abroad. Tesis doctoral. Universidad de Pennsylvania, Filadelfia.

SANJEK, ROGER (ed.). 1990. Fieldnotes. The Makings of Anthropology. Ithaca: Cornell University Press.

STOCKING, GEORGE W. (ed.). 1983. Observers Observed: Essays on Ethnographic Fieldwork. Madison: University of Wisconsin Press.

- 1992. The Ethnographer's Magic. Madison: University of Wisconsin Press.

Stoeltje, Beverly J.; Christie L. FoX y Stephen Olbrys. 1999. "The Self in 'Fieldwork'. A Methodological Concern". Journal of American Folklore 112 (444): 158-182.

VELASO, HONORIO y ÁNGel DíAz DE RADA. 1997. La lógica de la investigación etnográfica. Madrid: Trotta. 